\title{
Les procédures d'économicité sont à améliorer
}

Commençons par poser le cadre! La grande majorité des factures émises par les médecins, les pharmaciens, les hôpitaux etc. est payée par de l'argent «public», en particulier par l'assurance maladie obligatoire (19 milliards de francs par an) ou par l'impôt (9 milliards de francs). Comme chaque citoyen est obligé de payer primes de caisse-maladie et impôts, il ne s'agit pas d'un libre choix mais d'un acte de solidarité, et c'est donc la collectivité qui doit veiller à ce que cet argent soit dépensé de manière correcte: elle l'a fait en acceptant le 4 décembre 1994 la loi sur l'assurance-maladie.

Cette loi dit que l'argent "collectif» est dépensé de manière correcte s'il sert à rémunérer des prestations médicales qui sont efficaces, appropriées et économiques (art. 32 LAMal). Le troisième de ces critères - l'économicité - est précisé à l'art. 56: «Le fournisseur de prestations doit limiter ses prestations à la mesure exigée par l'intérêt de l'assuré et le but du traitement.»

Or c'est bien ce troisième critère qui anime tant de discussions aujourd'hui.

Sur le principe nous sommes tous d'accord. Mais il n'est pas toujours simple de définir l'intérêt de l'assuré, qui varie évidemment dans le temps et selon son état de santé. Et le but même du traitement n'est pas toujours clair: médecin, patient, assuré, assureur et citoyen peuvent légitimement avoir des avis différents sur un même cas de figure, tout simplement parce que leur optique est différente.

Le problème est que ces divergences sont en train de créer passablement de problèmes dans toute la Suisse: dans plusieurs cantons médecins et assureurs peinent à établir un climat de confiance menant à des solutions acceptables. Ces conflits pourraient avoir des conséquences néfastes pour des patients qui se verraient refuser la prescription de médicaments ou d'examens de laboratoire, ou encore des investigations radiologiques, pour éviter au médecin d'alourdir ses frais. Le médecin pourrait alors risquer de soigner davantage ses statistiques que ses patients, son souci devenant de ne pas avoir d'ennuis avec les caisses ni de devoir leur rembourser de l'argent.

Lorsqu'un médecin est soupçonné de ne pas respecter les critères d'économicité, il doit pouvoir se justifier: pour ce faire, il doit avoir confiance dans les institutions qui le jugent et dans la méthode du jugement. Or, et c'est bien là le problème, la confiance est trop souvent rompue, d'autant qu'aucun consensus n'existe sur les méthodes d'évaluation.
Cette situation n'est maintenant plus tolérable. C'est pourquoi la Commission de la sécurité sociale et de la santé du Conseil National a largement soutenu le 17 octobre trois initiatives parlementaires (07.483 Heim, 07.484 Meyer, 07.485 Cassis), dont le but est de modifier la LAMal et de garantir ainsi la sécurité juridique et la confiance nécessaires à résoudre ce problème. Ces initiatives veulent préciser les responsabilités des partenaires tarifaires, définir les critères essentiels quant à la méthode*, et fixer les délais pour trouver une solution partenariale. Le cas échéant, en l'absence d'accord, ce sera au Conseil fédéral de trancher.

Il s'agit finalement de donner l'occasion aux partenaires tarifaires, prestataires de soins et assureurs, d'assumer leur responsabilités dans l'intérêt des citoyens. Il s'agira aussi pour eux de réaliser que l'incapacité à trouver des solutions viables conduirait, comme dans le domaine tarifaire, à l'intervention directe de l'Etat - dont nous souhaiterions évidemment tous qu'il garde un rôle subsidiaire.

Ces initiatives parlementaires devront également être présentées à la Commission du Conseil des Etats: si nous réussissons à convaincre les sénateurs, nous pourrions avoir réglé la question en 2009 déjà. Mais cela dit, même la meilleure loi ne pourra atteindre son but sans notre ouverture à tous ni la volonté de gagner ensemble.

\section{Dr Ignazio Cassis, $\mathrm{MPH}$, vice-président de la FMH \\ Dr Jacques de Haller, président de la FMH}

Hanspeter Kuhn, avocat, responsable du service juridique de la FMH, secrétaire général adjoint de la FMH

\footnotetext{
* Par un changement de jurisprudence qu'il a introduit le 9 octobre 2006 (K 6/06), le Tribunal fédéral exige lui aussi la prise en compte des coûts totaux. Selon ce que nous comprenons des connaissances actuelles en la matière au plan international, cela signifie ceci: les statistiques doivent recenser adéquatement l'éventail d'activités du médecin (provider specialty definition); elles doivent inclure le degré de sévérité des maladies (case-mix/ severity of illness); et finalement, il faut qu'elles reflètent véritablement le cas et l'ensemble des ressources mobilisées pour lui (episodes of illness; line up all the health care resources into a defined event). La présente statistique de santésuisse ne correspond sur aucun de ces trois points à l'état actuel des connaissances, respectivement des exigences.
} 